\title{
Rational Enzyme Engineering Through Biophysical and Biochemical Modeling
}

Sandra Acebes ${ }^{1}$,Elena Fernandez-Fueyo ${ }^{2}$, Emanuele Monza ${ }^{1}$, M. Fatima Lucas ${ }^{1,3}$, David Almendral ${ }^{2}$ Francisco J. Ruiz-Dueñas ${ }^{2}$, Henrik Lund ${ }^{4}$, Angel T. Martinez ${ }^{2}$ and Victor Guallar ${ }^{1,5^{*}}$

${ }^{1}$ Joint BSC-CRG-IRB Research Program in Computational Biology, Barcelona Supercomputing Center, Jordi Girona 29, E-08034 Barcelona, Spain

${ }^{2}$ Centro de Investigaciones Biológicas, CSIC, Ramiro de Maeztu 9, E-28040 Madrid, Spain

${ }^{3}$ Anaxomics Biotech, Balmes 89, 08008 Barcelona, Spain

${ }^{4}$ Novozymes A/S, Krogshoejvej 36, DK-2880 Bagsvaerd, Denmark

${ }^{5}$ ICREA, Passeig Lluís Companys 23, E-08010 Barcelona, Spain

\section{Corresponding Author}

*Victor Guallar, Barcelona Supercomputing Center, Jordi Girona 29, E-08034 Barcelona, Spain. Phone: +34934137727. E-mail: victor.guallar@bsc.es

Keywords: Protein engineering $\bullet \operatorname{PELE} \bullet$ Oxidases $\bullet$ manganese peroxidase $\bullet$ enzyme design 
ABSTRACT: Due to its importance in the pharmaceutical industry, ligand dynamic simulations have experienced a great expansion. Using all-atom models and cutting edge hardware, one can perform non-biased ligand migration, active site search and binding studies. In this letter we demonstrate (and validate by PCR mutagenesis) how these techniques, when combined with quantum mechanics, open new possibilities in enzyme engineering. We provide a complete analysis where: 1) biophysical simulations produce ligand diffusion and, 2) biochemical modeling samples the chemical event. Using such broad analysis we engineer a highly stable peroxidase activating the enzyme for new substrate oxidation after rational mutation of two non-conserved surface residues. In particular, we create a new surface-binding site, quantitatively predicting the in vitro change in oxidation rate obtained by mutagenic PCR and achieving a comparable specificity constant to active peroxidases.

Nowadays, industry sectors demand efficient, cost-effective and sustainable processes and here enzymes pose a promising solution ${ }^{1}$. Industrial enzymes, however, often require additional engineering to increase stability and activity, and to turn off/on specificity. Rational mutagenesis based on mechanistic information, together with directed evolution, have centered this engineering labor ${ }^{2}$. In obtaining mechanistic information, computational studies based on bioinformatics ${ }^{3}$, molecular modeling (quantum and classical simulations), ${ }^{4}$ or de novo $\operatorname{design}^{5}$ (including combinations with directed evolution $^{6}$ ) are gaining importance. This information, however, has been typically limited to the active site. In these regards, rational engineering should aim for a complete description of the enzymatic process: 1) substrate migration and recognition, typically studied by biophysical techniques, and 2) the biochemical reaction, modeled by quantum chemistry methods.

Characterizing ligand diffusion with biophysical modeling has experienced an extraordinary advance. Motivated by the pharmacological industry, hardware and software development allow today a description of unbiased ligand migration and binding. Most of these developments focused on molecular dynamics algorithms, where microsecond simulations have been reported for several 
systems $^{7}$. In addition, Monte Carlo studies using PELE (Protein Energy Landscape Exploration) software have produced analogous results ${ }^{8}$. Regarding the biochemical study, mixed quantum mechanics/molecular mechanics $(\mathrm{QM} / \mathrm{MM})$ techniques are routinely performed in complex systems ${ }^{9}$ which, together with improvement in electronic coupling methodologies, allow for accurate characterization of oxidation and electron transfer processes ${ }^{10}$. Combining such advances in biophysical and biochemical modeling techniques, one can map in detail an enzymatic mechanism ${ }^{11}$ and, as we show here, turn this extensive knowledge into enzymatic rational design. In particular, we convert MnP6, a 2,2'-azino-bis(3-ethylbenzothiazoline-6-sulfonic acid) (ABTS) inactive but highly stable at low $\mathrm{pH}$ manganese peroxidase (MnP) from Ceriporiopsis subvermispora into an active one. To this aim, we also study MnP4, a less stable (at low pH) but ABTS-oxidyzing MnP from Pleurotus ostreatus ${ }^{12}$.

MnPs are oxidoreductases with industrial interest capable of replacing harsh chemicals in oxidation processes, in delignification/bleaching of wood pulp or degradation of xenobiotic compounds. As in other peroxidases, MnPs have different binding sites. One site, as shown by crystallography ${ }^{13}$, is in contact with the heme propionate (Figure 1A). The other one, known as the main heme channel, is located in the heme edge at the distal histidine side. At first sight, the main difference between MnP4 and MnP6 (with 46\% sequence identity and 57\% sequence similarity) is the C-terminal tail: 29 extra residues in MnP6 block the propionate channel (Figure 1A), deletion of which introduces ABTS oxidation in this enzyme at the expense of thermostability decrease $\mathrm{e}^{14}$.
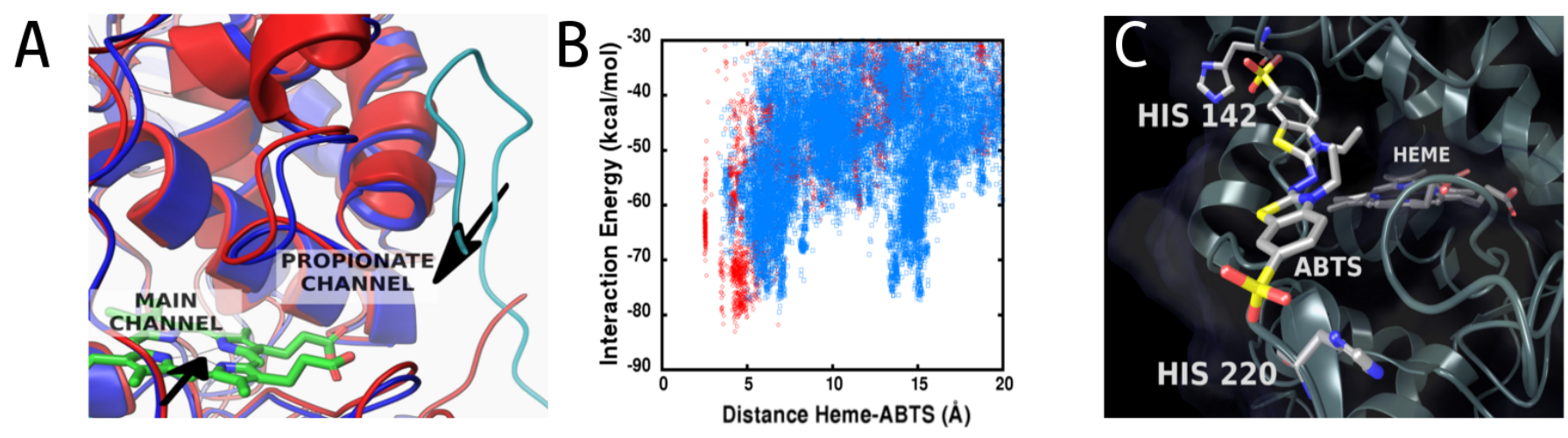
Figure 1. (A) MnP4 (red) and MnP6 (blue) superposition; extra C-terminal residues in MnP6 are highlighted in light blue. (B) Interaction energy obtained from PELE for ABTS diffusion in MnP4 (red) and MnP6 (blue); distances involve the lowest distance between any two atoms from each molecule. (C) ABTS bound structure corresponding to the lowest interaction energy in MnP4's main channel.

To begin with the rational engineering study, substrate diffusion and active site search was performed by running the 'unconstrainded ligand exploration and binding site search' with PELE on both enzymes. This task can be run freely using the ready made script with the quoted name above at https://pele.bsc.es $^{15}$. Figure 1B shows the differences in ABTS diffusion between MnP4 and MnP6. Clearly, MnP4 presents better interaction energies in close proximity to the heme group. As mentioned, lack of oxidation in MnP6 has been rationalized by the extra C-terminal residues, and ABTS oxidation in $\mathrm{MnP} 4$ has been attributed to the larger opening in the propionates ${ }^{14}$. The substrate migration, however, reveals the best minimum in MnP4 placed at the main heme channel (Figure 1C, see also the supporting video), with a second minimum ( $\sim 7 \mathrm{kcal} / \mathrm{mol}$ higher in energy) located at the propionate channel. Even more surprising was the fact that in MnP6 the best bound structures are found in vicinity of the propionate channel, although significantly further away than the structures in MnP4 due to the (extra residues) C-terminal steric hindrance. In MnP6, we find no low energy poses in the main channel as the one observed in MnP4.

Table 1. Electronic coupling values (eV).

\begin{tabular}{ccc} 
& $\begin{array}{c}\text { Propionate } \\
\text { Channel }\end{array}$ & $\begin{array}{c}\text { Heme } \\
\text { Channel }\end{array}$ \\
\hline MnP4 & $0.27 \mathrm{E}-3$ & $0.70 \mathrm{E}-2$ \\
MnP6 & $0.46 \mathrm{E}-5$ & - \\
MnP6 $(\mathrm{G} 139 \mathrm{H} / \mathrm{N} 218 \mathrm{H})$ & $0.46 \mathrm{E}-5$ & $0.18 \mathrm{E}-2$ \\
\hline
\end{tabular}


Following the biophysical study, we performed the quantum (bio)chemistry characterization of the electronic coupling involved in the oxidation process. In MnP4, the best minimum at the heme channel shows $25 \mathrm{x}$ better electronic coupling $(0.70 \mathrm{E}-2$ vs $0.27 \mathrm{E}-3 \mathrm{eV})$ than the local minimum at the propionate channel (Table 1). While interaction energies and electronic coupling values are good for both oxidation sites, i.e. MnP4 most likely oxidizes ABTS at both sites, values at the main channel are significantly better. In contrast, the electronic coupling for MnP6 is drastically lower, $0.46 \mathrm{E}-5 \mathrm{eV}$, as a result of the worst substrate binding and in agreement with lack of oxidation.

Inspecting the minimum in MnP4's main heme channel (Figure 1C), we find two histidines, H220 and H142, interacting with ABTS's negatively charged sulfonates, forming $1.5 \AA$ and $1.8 \AA$ hydrogen bond (based on optimal $\mathrm{pH}$ for $\mathrm{MnP} 4$ activity, 3.5, these histidines were protonated). Interestingly, these histidines are not found in MnP6 (finding G139 and N218 instead) or in any long MnP sequences analyzed (Figure 2B), while they are present in a few short MnPs (Figure 2A). Moreover, they are located away from the active site and solvent exposed, making them not a primary choice in mutation studies. For example, both residues do not present a high mutability score in HotSpotWizard (http://loschmidt.chemi.muni.cz/hotspotwizard, default options with no predefined active site), a bioinformatics tool for detecting hot spots for engineering substrate specificity, activity or enantioselectivity in enzymes.

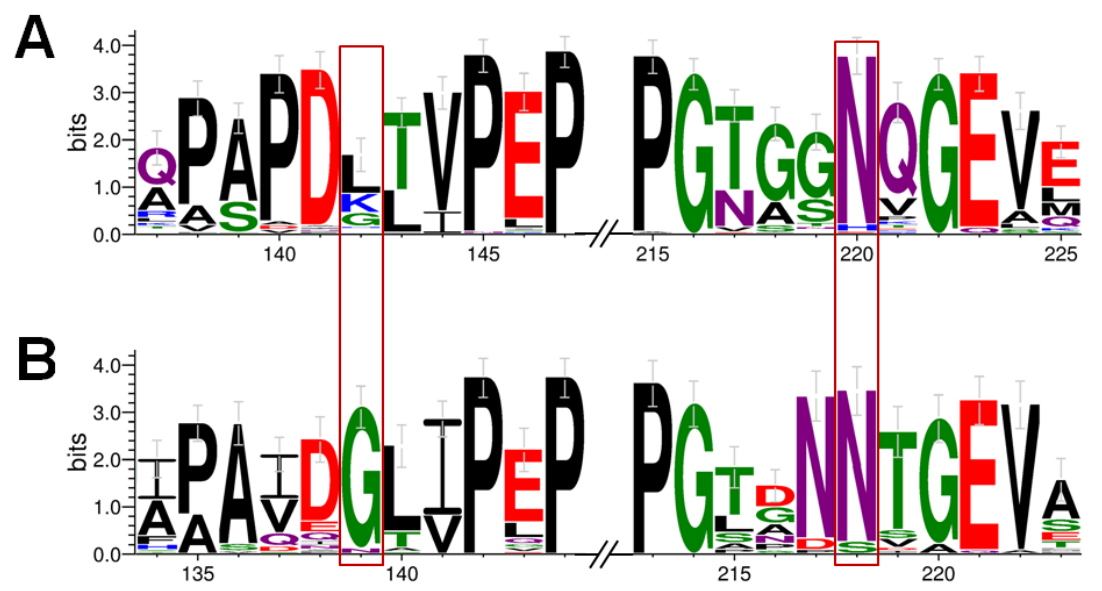

Figure 2. Sequence logo of residues surrounding long MnP6 Gly139 (left) and Asn218 (right) in 75 short MnPs (A) and 53 long MnPs (B) available from genomes and databases (from WebLogo 3.4). 
This detailed mechanistic knowledge opens new possibilities in engineering MnP6. Thus, we proceed with in silico $\mathrm{G} 139 \mathrm{H}$ and $\mathrm{N} 218 \mathrm{H}$ mutations and with a local (main channel) ligand exploration with PELE ("local refinement" script at https://pele.bsc.es ${ }^{15}$ ). Figure 3A shows the interaction energies for MnP4 and MnP6 wild type proteins. Due to the enhancement in the local sampling, where the ligand is restrained to a $12 \AA$ distance from the heme-CHB atom, ABTS interaction energies in MnP4 slightly improve from the previous full surface exploration. MnP6 energies, however, do not show again any significant minima, confirming the lack of substrate oxidation at this site. This picture completely changes in the in silico engineered MnP6 double mutant (Figure 3B), where energy and distance values are now similar to the MnP4 ones. Thus, by mutating two non-conserved surface residues, we created a surface binding site in MnP6.
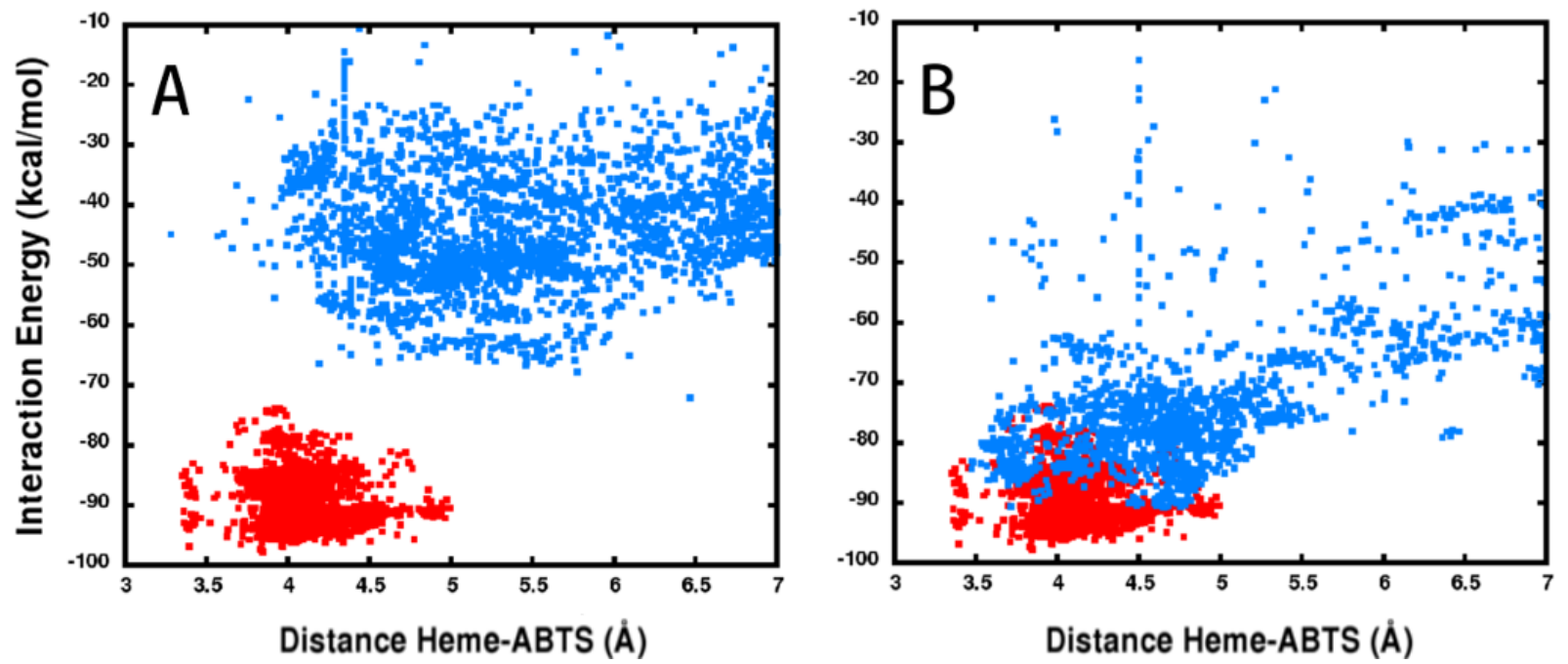

Figure 3. (A, B) Interaction energy for ABTS binding in the main channel for MnP4 (red dots), wild type MnP6 (panel A, blue dots) and double mutant (panel B, blue dots) obtained from the local ABTS diffusion.

Figure 4 shows the main channel best binding energy structure for MnP4 and MnP6 mutant, clearly adopting similar binding modes. Biochemical simulation of this new minimum in MnP6's double mutant shows an electronic coupling value only $\sim 4$ times lower than the one in MnP4, 0.18E-2 vs 
$0.70 \mathrm{E}-2 \mathrm{eV}$, respectively. This is almost a three orders of magnitude increase from the wild type MnP6 value, whose best (non-reactive) position was found in the propionate channel.
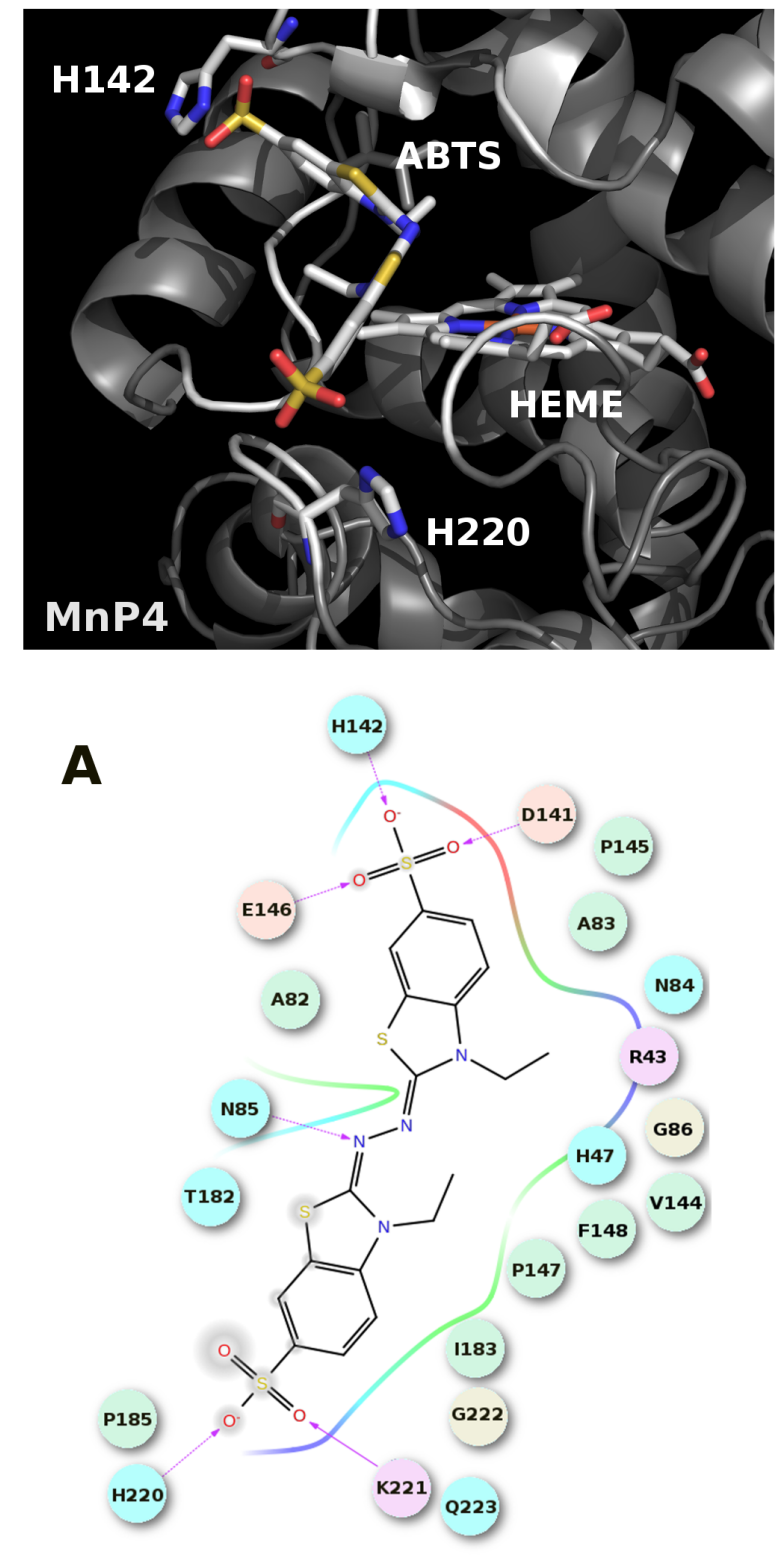
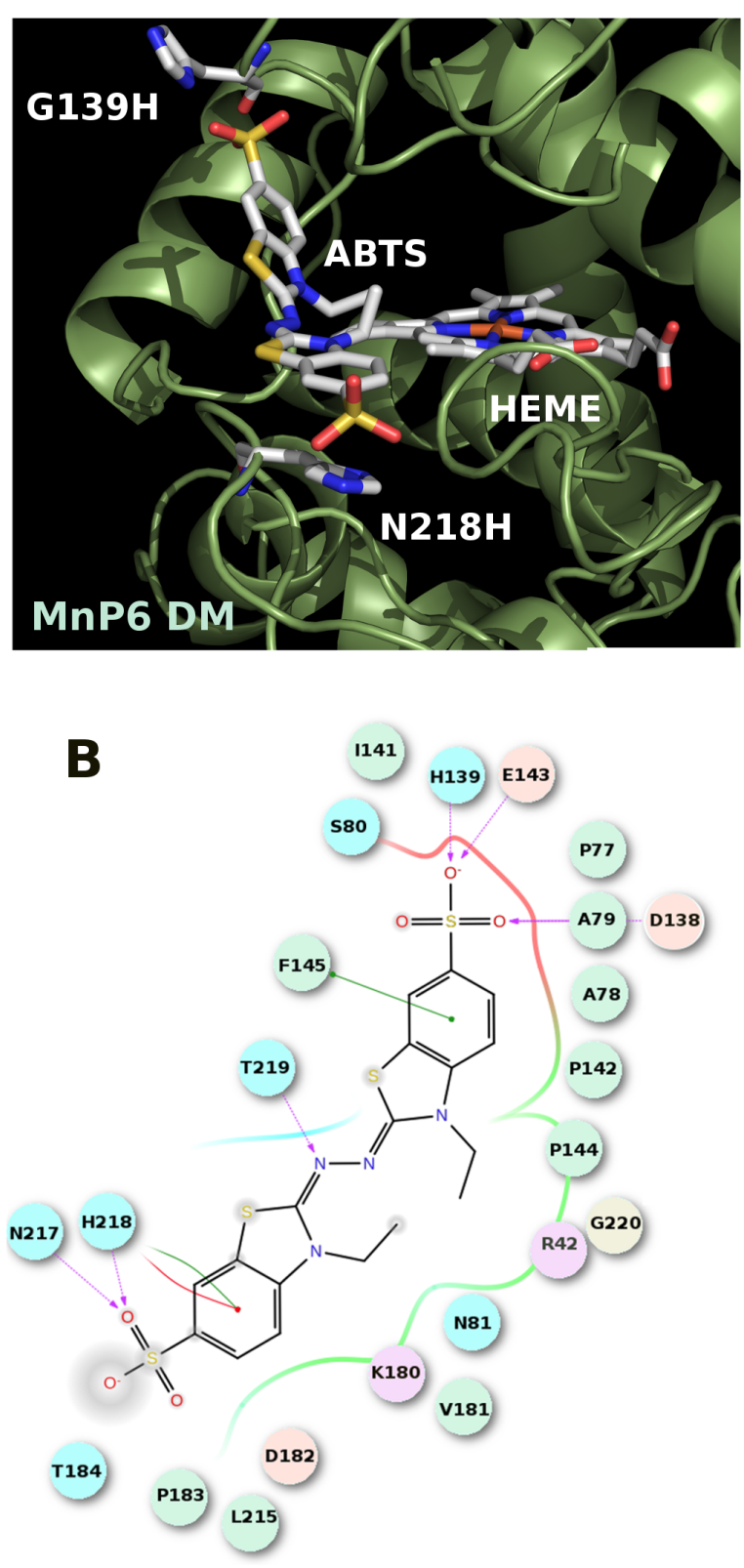

Figure 4. ABTS best binding energy structures for MnP4 (A) and MnP6 double mutant (B) in the main channel. $3 \mathrm{D}$ models are shown above and $2 \mathrm{D}$ projections, with detail interactions, immediately below. 
Finally, the double mutant was produced by mutagenic PCR. Enzyme kinetic properties are shown in Table 2; the hyperbolic fits used to calculate the steady-state kinetic constants are also shown in Figure S2. As predicted, the mutant is now able to successfully oxidize ABTS, despite the access to the heme propionate is still blocked by the $\mathrm{C}$-terminal tail ${ }^{14}$, due to the new engineered site at the main heme access channel. Interestingly, the 13.7 fold decrease in turnover rate $\left(k_{\text {cat }}\right)$ between MnP4 and MnP6 double mutant, agrees with the $\sim 4$ times (3.8 to be precise) predicted reduction in electronic coupling (table 1). Considering Marcus theory, the rate is proportional to the square power of the electronic coupling: a 3.8 fold decrease in electronic coupling would correspond to a 14.4 fold decrease in ET rate. Importantly, the two mutations introduced here only slightly diminished the extreme acidic-pH stability of C. subvermispora MnP6, from $93 \%$ to $80 \%$ residual activity after $4 \mathrm{~h}$ at $\mathrm{pH} 2$, compared with most ligninolytic peroxidases, including P. ostreatus $\mathrm{MnP} 4$ that is immediately $(<1 \mathrm{~min})$ inactivated at $\mathrm{pH}$ $2^{12}$. The possibility to catalyze oxidations at acidic $\mathrm{pH}$, strongly increases the biotechnological interest of peroxidases since the redox potential of the heme cofactor increases when the $\mathrm{pH}$ decreases.

Table 2. ABTS oxidation kinetic constants for MnP4, MnP6 and MnP6 double mutant (DM, G139H/N218H)

\begin{tabular}{cccc}
\hline & MnP4 & MnP6 & MnP6 DM \\
\hline$K_{\mathrm{m}}(\mu \mathrm{M})$ & $1560 \pm 76$ & - & $284 \pm 30$ \\
$k_{\mathrm{cat}}\left(\mathrm{s}^{-1}\right)$ & $128 \pm 3$ & - & $9.3 \pm 0.3$ \\
$k_{\mathrm{cat}} / K_{\mathrm{m}}\left(\mathrm{s}^{-1} \mathrm{mM}^{-1}\right)$ & $82 \pm 3$ & - & $33 \pm 3$ \\
\hline
\end{tabular}

In conclusion, developments in ligand diffusion simulations, when combined with quantum chemistry, provide a robust description of the biophysical and biochemical processes in enzymatic catalysis. With such detailed description, we were able to perform MnP6's engineering, which was confirmed by in vitro production and characterization. In this way, we turned an ABTS inactive but 
highly stable at acidic pH peroxidase, into an enzyme with new oxidizing capabilities by introducing two specific surface mutations far away from the active site. Overall, the capability of conducting such detailed studies underlines the importance of computational modeling in future enzymatic biotechnology.

\section{METHODS}

System setup. Crystal structures 4BM1 and 4CZN were selected for MnP4 and MnP6, respectively. Systems were prepared with the Protein Preparation Wizard ${ }^{16}$, fixing environment dependent protonation states. Heme and ABTS molecular mechanics parameters were obtained from a QM optimization of the system at the unrestricted DFT functional M06 ${ }^{17,18}$ and lacvp* basis set level of theory.

Substrate diffusion with PELE (Protein Energy Landscape Exploration). Substrate diffusion and docking was modeled with PELE, ${ }^{15}$ a Monte Carlo based algorithm that produces putative new configurations through a sequential ligand and protein perturbation scheme, side chain prediction and minimization steps. For the full surface exploration ABTS was manually placed in 20 initial random positions on the protein's surface. Ligand perturbation was performed with random translations (6 and 1 $\AA$ A) and rotations $(0.25$ and $0.02 \mathrm{rad})$. Protein perturbation was based on the lowest 6 anisotropic normal $\operatorname{modes}^{19}$. Side chain sampling included all residues within $5 \AA$ from the ligand. Finally, the global minimization optimizes the new configuration, which is filtered with a Metropolis acceptance test, where the energy is described with an all-atom OPLS force field with a surface generalized Born solvent model.Global search results were based on 120 trajectories (1 trajectory per computing core) x $48 \mathrm{~h}$ simulations. Enhanced local sampling was obtained by running 120 trajectories x $24 \mathrm{~h}$, where the ligand center of mass is constrained within $12 \AA$ from the heme- $C H B$ atom. PELE's interaction energies indicate the internal energy difference between the complex and the free protein and ligand $(\mathrm{AB}-\mathrm{A}$ - 
B). These energies have significant larger values than binding free energies, although its qualitative analysis (when comparing similar binding modes) is analogous.

Electronic coupling calculations. From best binding energy structures obtained in PELE's explorations, ecoupling calculations were performed using the e-coupling server (http://ecouplingserver.bsc.es). First, systems were optimized at the QM/MM level where the heme, distal and proximal histidines and ABTS were included in the QM region. The rest of the protein and solvent were in the MM region. Solvation was accomplished by adding explicit waters and running 0.5 ns with Desmond (default protocol) where all heavy atoms where frozen. All QM/MM calculations were performed with Qsite ${ }^{20}$ using the M06-L(lacvp*)/OPLS level of theory. Electronic couplings were computed using the Fragment Charge Difference Method (FCD) ${ }^{21}$ which calculates the coupling between donor and acceptor by unitary transformation of adiabatic states to diabatic states, using as operator the charge difference between donor and acceptor.

Gene synthesis and heterologous expression. The coding sequences of the $C$. subvermispora gene model encoding isoenzyme MnP6 (50686) and of P. ostreatus gene model encoding isoenzyme MnP4 (1099081) were designed with E. coli preferred codon usage (confirmed for other genes expressed in the BL21 strain), and synthesized by ATG:biosynthetics. The two genes were cloned in the expression vector pET23a $(+)$ (Novagen). The resulting plasmids - pET23a-50686 and pET23a-1099081 - were used for expression and directed mutagenesis. E. coli DH5 $\alpha$ was used for plasmid propagation, and peroxidases were produced in E. coli BL21 (DE3) pLys, in vitro folded and purified as previously described..$^{12,22}$

Directed mutagenesis. G139H and N218H mutations were introduced in the MnP6 encoding gene by PCR (polymerase chain reaction) using the expression plasmid pET23a-50686 as template. The 5'- CGC GGCT ATC GAT CAT CTT ATT CCC GAG CCC C -3' and 5'- CCC CGG ACT CGA CAA CAC ACC GGT GAG GTC TCG TCG CCG C -3' direct primer (and the reverse primer bearing the complementary sequence) were used, respectively. 
Kinetics of ABTS oxidation and pH stability. Absorbance changes during substrate oxidation in $0.1 \mathrm{M}$ tartrate $\mathrm{pH} 3.5$ (optimal $\mathrm{pH}$ ) were recorded in a UV-160 spectrophotometer (Shimadzu). ABTS oxidation was followed from cation radical $\left(\varepsilon_{436} 29.3 \mathrm{mM}^{-1} \cdot \mathrm{cm}^{-1}\right)$ formation. All reactions were carried out at $25^{\circ} \mathrm{C}$ (using $\sim 0.01 \mu \mathrm{M}$ enzyme) and initiated by addition of $0.1 \mathrm{mM} \mathrm{H}_{2} \mathrm{O}_{2}$. Means and standard errors for $K_{\mathrm{m}}$ and $k_{\text {cat }}$ were obtained by nonlinear least-squares fitting to the Michaelis- Menten model. Fitting of these constants to the normalized equation $v=\left(k_{\text {cat }} / K_{\mathrm{m}}\right)[\mathrm{S}] /\left(1+[\mathrm{S}] / K_{\mathrm{m}}\right)$, where $v$ is the reaction rate and $\mathrm{S}$ is the substrate concentration, yielded the catalytic efficiency values $\left(k_{\mathrm{cat}} / K_{\mathrm{m}}\right)$ with their corresponding standard errors. The $\mathrm{pH}$ stability was estimated after pre-incubating the enzyme in the range of $\mathrm{pH} 2-8$. Residual activities were estimated immediately after mixing, and after 1 and $4 \mathrm{~h}$ incubation at $4^{\circ} \mathrm{C}$. 


\section{ASSOCIATED CONTENT}

Supporting Information. A video illustrating the substrate's exploration, the multiple sequence alignment of $\mathrm{MnPs}$ from $P$. ostreatus and $C$. subvermispora genomes, and the hyperbolic fits used to calculate the steady-state kinetic constants. This material is available free of charge via the Internet at http://pubs.acs.org.

\section{AUTHOR INFORMATION}

Corresponding Author

*Email: victor.guallar@bsc.es

\section{ACKNOWLEDGMENT}

This work was supported by the INDOX (KBBE-2013-7-613549 to ATM) European project, and the CTQ2013-48287 (to VG) and BIO2014-56388-R (to FJR-D) projects of the Spanish Ministry of Economy and Competitiveness (MINECO). FJR-D acknowledges a MINECO Ramón\&Cajal contract.

\section{REFERENCES}

1. Schafer, T.; Borchert, T. W.; Nielsen, V. S.; Skagerlind, P.; Gibson, K.; Wenger, K.; Hatzack, F.; Nilsson, L. D.; Salmons, S.; Pedersen, S.; Heldt-Hansen, H. P.; Poulsen, P. B.; Lund, H.; Oxenbøll, K. M.; Wu, G. F.; H.H., P.; Xu, H., Industrial Enzymes. In White Biotechnology, Ulber, R.; Sell, D., Eds. Springer: Berlin, 2007; pp 59-131.

2. (a) Cherry, J. R.; Lamsa, M. H.; Schneider, P.; Vind, J.; Svendsen, A.; Jones, A.; Pedersen, A. H., Directed evolution of a fungal peroxidase. Nat. Biotechnol. 1999, 17 (4), 379- 
384; (b) Savile, C. K.; Janey, J. M.; Mundorff, E. C.; Moore, J. C.; Tam, S.; Jarvis, W. R.; Colbeck, J. C.; Krebber, A.; Fleitz, F. J.; Brands, J.; Devine, P. N.; Huisman, G. W.; Hughes, G. J., Biocatalytic Asymmetric Synthesis of Chiral Amines from Ketones Applied to Sitagliptin Manufacture. Science 2010, 329 (5989), 305-309.

3. Yang, J.-S.; Seo, S. W.; Jang, S.; Jung, G. Y.; Kim, S., Rational Engineering of Enzyme Allosteric Regulation through Sequence Evolution Analysis. PLoS Comput. Biol. 2012, 8 (7), e1002612.

4. (a) Privett, H. K.; Kiss, G.; Lee, T. M.; Blomberg, R.; Chica, R. A.; Thomas, L. M.; Hilvert, D.; Houk, K. N.; Mayo, S. L., Iterative approach to computational enzyme design. Proc. Natl. Acad. Sci. U. S. A. 2012, 109 (10), 3790-3795; (b) Grisewood, M. J.; Gifford, N. P.; Pantazes, R. J.; Li, Y.; Cirino, P. C.; Janik, M. J.; Maranas, C. D., OptZyme: Computational Enzyme Redesign Using Transition State Analogues. PLoS ONE 2013, 8 (10), e75358; (c) Osuna, S.; Jiménez-Osés, G.; Noey, E. L.; Houk, K., Molecular Dynamics Explorations of Active Site Structure in Designed and Evolved Enzymes. Acc. Chem. Res. 2015, 48 (4), 10801089; (d) Narayan, A. R.; Jiménez-Osés, G.; Liu, P.; Negretti, S.; Zhao, W.; Gilbert, M. M.; Ramabhadran, R. O.; Yang, Y.-F.; Furan, L. R.; Li, Z., Enzymatic hydroxylation of an unactivated methylene $\mathrm{C}-\mathrm{H}$ bond guided by molecular dynamics simulations. Nat. Chem. 2015, 7 (8), 653-660.

5. $\quad$ (a) Siegel, J. B.; Zanghellini, A.; Lovick, H. M.; Kiss, G.; Lambert, A. R.; St.Clair, J. L.; Gallaher, J. L.; Hilvert, D.; Gelb, M. H.; Stoddard, B. L.; Houk, K. N.; Michael, F. E.; Baker, D., Computational Design of an Enzyme Catalyst for a Stereoselective Bimolecular Diels-Alder Reaction. Science 2010, 329 (5989), 309-313; (b) Eiben, C. B.; Siegel, J. B.; Bale, J. B.; Cooper, S.; Khatib, F.; Shen, B. W.; Players, F.; Stoddard, B. L.; Popovic, Z.; Baker, D., Increased DielsAlderase activity through backbone remodeling guided by Foldit players. Nat. Biotechnol. 2012, 30 (2), 190-192.

6. Khersonsky, O.; Kiss, G.; Röthlisberger, D.; Dym, O.; Albeck, S.; Houk, K. N.; Baker, D.; Tawfik, D. S., Bridging the gaps in design methodologies by evolutionary optimization of the stability and proficiency of designed Kemp eliminase KE59. Proc. Natl. Acad. Sci. U. S. A. 2012, 109 (26), 10358-10363.

7. (a) Buch, I.; Giorgino, T.; De Fabritiis, G., Complete reconstruction of an enzymeinhibitor binding process by molecular dynamics simulations. Proc. Natl. Acad. Sci. U. S. A.

2011, 108 (25), 10184-10189; (b) Shan, Y.; Kim, E. T.; Eastwood, M. P.; Dror, R. O.; Seeliger, M. A.; Shaw, D. E., How Does a Drug Molecule Find Its Target Binding Site? J. Am. Chem. Soc. 2011, 133, 9181-9183.

8. Kotev, M.; Lecina, D.; Tarrago, T.; Giralt, E.; Guallar, V., Unveiling prolyl oligopeptidase ligand migration by comprehensive computational techniques. Biophys. J. 2015, $108(1), 116-25$.

9. van der Kamp, M. W.; Mulholland, A. J., Combined Quantum Mechanics/Molecular Mechanics (QM/MM) Methods in Computational Enzymology. Biochemistry 2013, 52 (16), 2708-2728.

10. (a) Wallrapp, F.; Voytiuk, A.; Guallar, V., In-silico Assessment of protein-protein electron transfer. A case study: Cytochrome c Peroxidase - cytochrome c. Plos Comput. Biol. 2013, 9, e1002990; (b) Prytkova, T. R.; Kurnikov, I. V.; Beratan, D. N., Coupling Coherence Distinguishes Structure Sensitivity in Protein Electron Transfer. Science 2007, 315 (5812), 622625. 
11. Monza, E.; Lucas, M. F.; Camarero, S.; Alejaldre, L. C.; Martínez, A. T.; Guallar, V., Insights into Laccase Engineering from Molecular Simulations: Toward a Binding-Focused Strategy. J. Phys. Chem. Lett. 2015, 6 (8), 1447-1453.

12. Fernandez-Fueyo, E.; Ruiz-Duenas, F.; Martinez, M.; Romero, A.; Hammel, K.; Medrano, F.; Martinez, A., Ligninolytic peroxidase genes in the oyster mushroom genome: heterologous expression, molecular structure, catalytic and stability properties, and lignindegrading ability. Biotechnol. Biofuels 2014, 7 (1), 2.

13. Sharp, K. H.; Mewies, M.; Moody, P. C. E.; Raven, E. L., Crystal structure of the ascorbate peroxidase-ascorbate complex. Nat. Struct. Mol. Biol. 2003, 10 (4), 303-307.

14. Fernandez-Fueyo, E.; Acebes, S.; Ruiz-Duenas, F. J.; Martinez, M. J.; Romero, A.; Medrano, F.; Guallar, V.; Martinez, A. T., Structural implications of the C-terminal tail in the catalytic and stability properties of manganese peroxidases from ligninolytic fungi. Acta Crystallogr. Sect. D 2014, 70, 3253-3265.

15. Madadkar-Sobhani, A.; Guallar, V., PELE web server: atomistic study of biomolecular systems at your fingertips. Nucleic Acids Res. 2013, 41 (W1), W322-W328.

16. Madhavi Sastry, G.; Adzhigirey, M.; Day, T.; Annabhimoju, R.; Sherman, W., Protein and ligand preparation: parameters, protocols, and influence on virtual screening enrichments. $J$. Comput. Aided Mol. Des. 2013, 27 (3), 221-234.

17. Zhao, Y.; Truhlar, D., The M06 suite of density functionals for main group thermochemistry, thermochemical kinetics, noncovalent interactions, excited states, and transition elements: two new functionals and systematic testing of four M06-class functionals and 12 other functionals. Theor. Chem. Acc. 2008, 120 (1-3), 215-241.

18. Zhao, Y.; Truhlar, D. G., Density functionals with broad applicability in chemistry. Acc. Chem. Res. 2008, 41 (2), 157-167.

19. Bahar, I.; Atilgan, A. R.; Erman, B., Direct evaluation of thermal fluctuations in proteins using a single-parameter harmonic potential. Folding \& Design 1997, 2 (3), 173-181.

20. Qsite QSite, version 6.1; Schrödinger, LLC: New York NY, 2013.

21. Voityuk, A. A.; Rösch, N., Fragment charge difference method for estimating donoracceptor electronic coupling: Application to DNA $\pi$-stacks $J$. Chem. Phys. 2002, 117, 5607 5616.

22. Fernández-Fueyo, E.; Ruiz-Dueñas, F. J.; Miki, Y.; Martínez, M. J.; Hammel, K. E.; Martínez, A. T., Lignin-degrading Peroxidases from Genome of Selective Ligninolytic Fungus Ceriporiopsis subvermispora. J. Biol. Chem. 2012, 287 (20), 16903-16916. 
TOC GRAPHIC
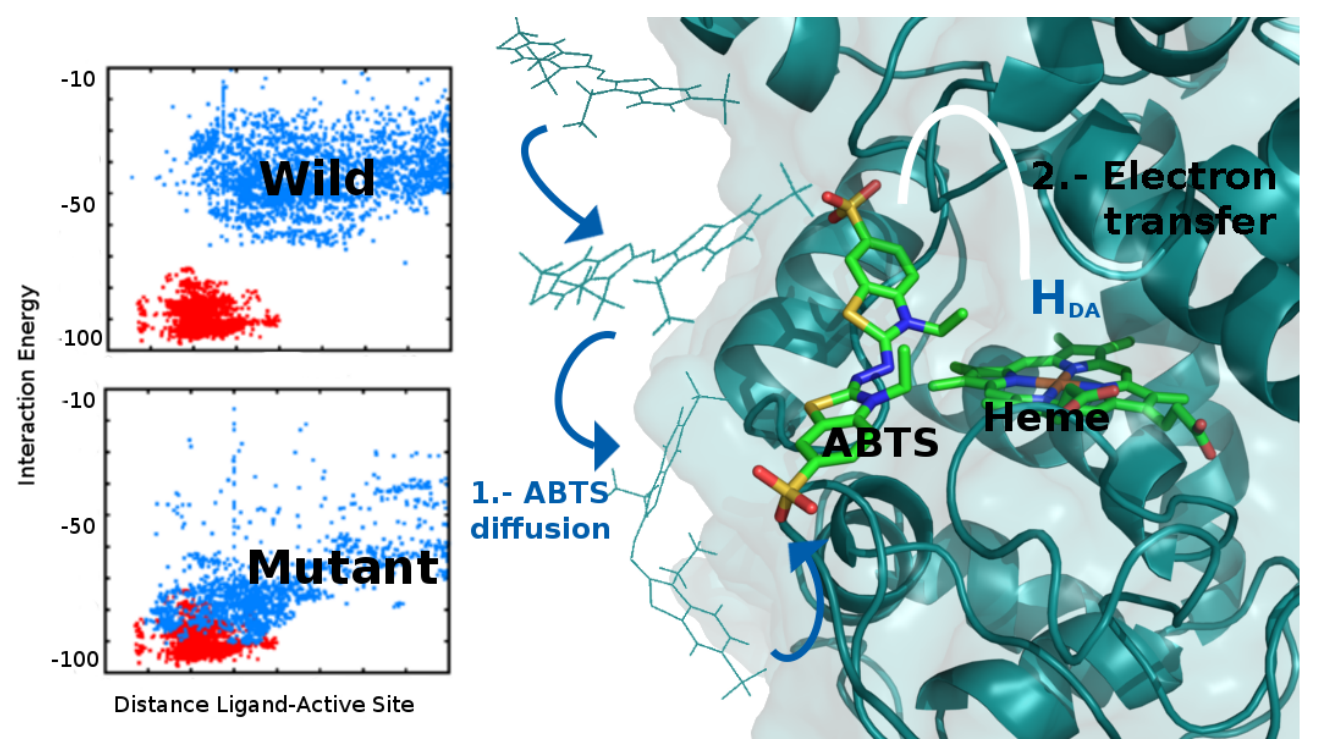